\title{
Review Paper: Routines in Families of Children With Autism
}

\author{
${ }^{*}$ Hooshang Mirzaie ${ }^{1} \odot$, Ehsan Jamshidian ${ }^{1} \odot$, Seyyed Ali Hosseini ${ }^{1}$
}

1. Department of Occupational Therapy, University of Social Welfare and Rehabilitation Sciences, Tehran, Iran.

\begin{tabular}{l|l}
$\begin{array}{l}\text { Use your device toscan } \\
\text { and read the article online }\end{array}$ \\
bilitation. 2018; 19(3):184-193. http://dx.doi.org/10.32598/rj.19.3.184 \\
dol: $:$ http://dx.doi.org/10.32598/rj.19.3.184
\end{tabular}

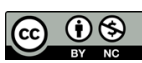

Funding: See Page 190

Received: 10 Feb 2018 Accepted: 18 Jun 2018 Available Online: 01 Oct 2018

Keywords: Autism, Family, Routines, Participation

\section{ABSTRACT}

Objective Routines are a set of daily and regular activities that play an important role in the development of the child and the family. These activities include building the structure for daily life, developing social skills, academic skills, speech skills, facilitating family relationships, and creating a sense of belonging among family members. This study examines the routines of families of children with autism.

Materials \& Methods Routines are a set of daily and regular activities that play an important role in the development of the child and the family. These activities include building the structure for daily life, developing social skills, academic skills, speech skills, facilitating family relationships, and creating a sense of belonging among family members. This study examines the routines of families of children with autism. Results Routines are related to family health. However, following routines in the families of children with autism are difficult due to factors associated with the child such as lack of flexibility, problematic behaviors and sensory-processing problems; factors associated with care givers, such as parents' anxiety or marital problems; and environmental factors such as the lack of access to autism healthcare services in less populated and remote areas. Thus, parents of children with autism face the challenge of creating and maintaining the routines. A child with autism usually takes the center of family structure and activities. This condition will eventually end in controlling the daily routines of families. In other words, a significant portion of the daily life of the family is linked to the needs of the child with autism. Some routines such as eating and sleeping have been specifically addressed as challenging routines. The structure of routines takes shape around the needs of the child, rather than the whole family, and in some cases, the needs of other family members are ignored. The presence of a child with autism in the family negatively affects the development of meaningful interactions. The pervasive nature of autism leads to inflexible, child-focused routines and limited emotional and social experiences during everyday life activities in their families. Despite the challenges that families of autistic children may have to participate in routines, these families try to engage in challenging routines, which have meaning and importance for them, to learn their child's endurance. They do not give a chance to the child with autism to decide for their family and collaborate with family members.

Conclusion Despite the important role of routines in the growth and health of the child and the family, it seems that the disabling nature of autism with its many complications, which overshadows other family planning affect the quantity and quality of family participation in activities and developing meaningful routines. Although the development of a child with autism has a burden on the family, inability to create interactions, understanding the child, and communicating among family members makes the situation in the family more problematic. As a result, the families of children with autism are unable to create and maintain effective routines for the optimal participation of family members that eventually leads to the loss of family integrity and solidarity.

\section{* Corresponding Author: \\ Hooshang Mirzaie, PhD}

Address: Department of Occupational Therapy, University of Social Welfare and Rehabilitation Sciences, Tehran, Iran.

Tel: +98 (21) 22180037

E-Mail: ho.mirzaie@uswr.ac.ir 


\title{
روالهاى رايج روزمره در خانوادههاى كودكان دار ایى اتيسم: مرور نظاممند
}

\author{
"هوشنگ ميرزايى' (1)، احسان جمشيديان' (1)، سيد على حسينى' \\ 1 - كروه كاردرمانى، دانشخاه علوم بهزيستى و توانبخشى، تهران، ايران.
}

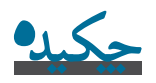

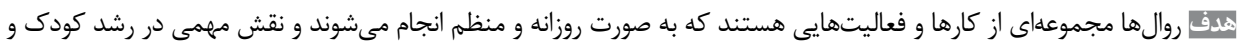

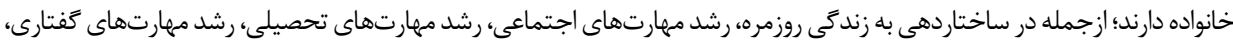

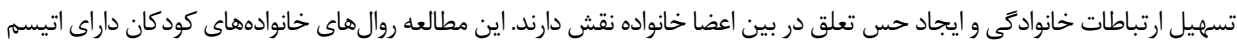
رابر سىى مى كند.

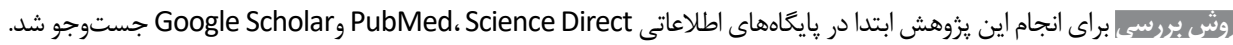

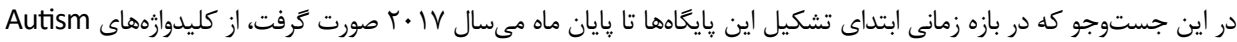

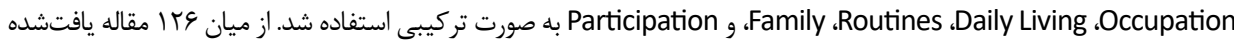

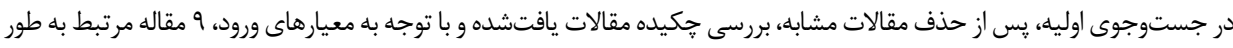

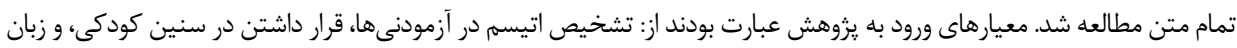
فارسى يا انخليسى مقاله.

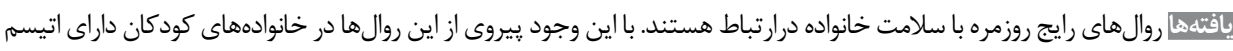

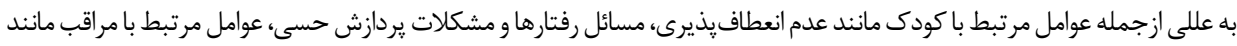

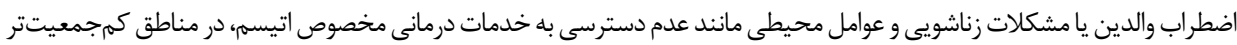

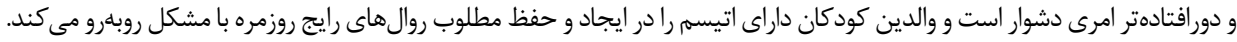

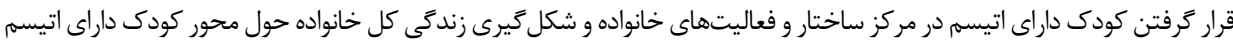

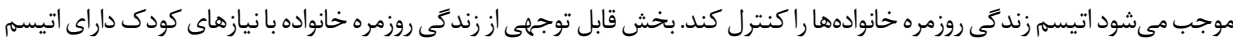

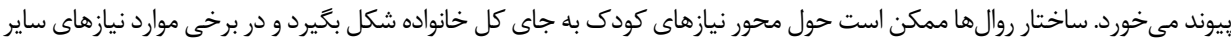

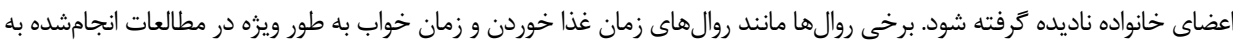

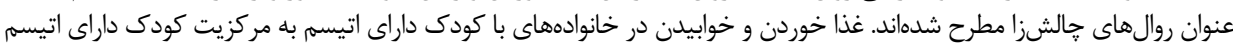

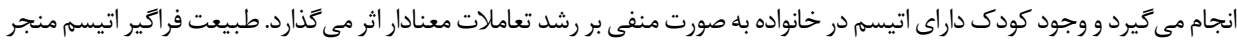

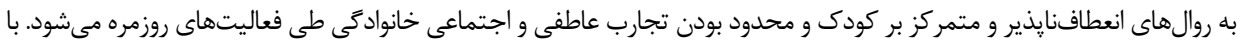

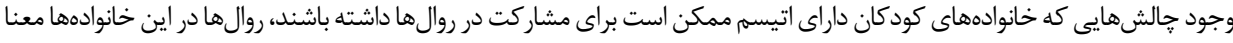

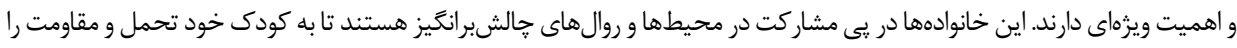

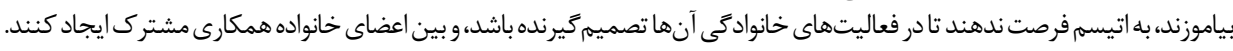

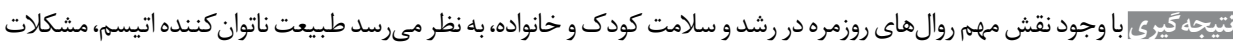

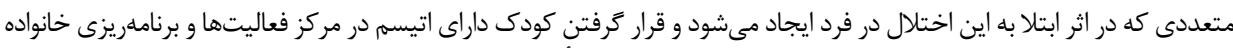

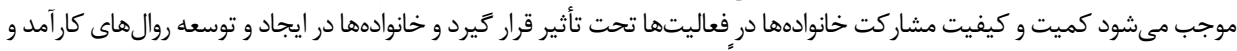

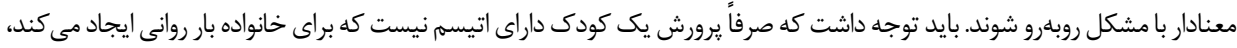

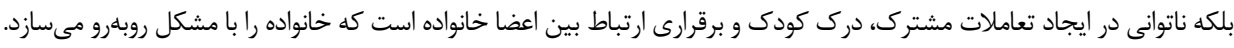

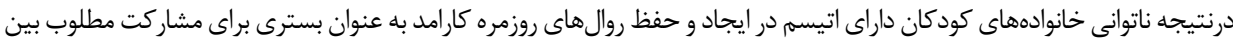

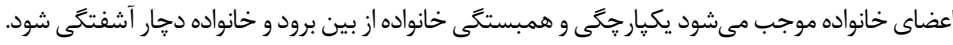

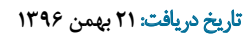

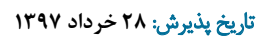

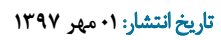

كليدوارهزها:

اتيسم، خانواده،

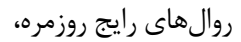
مشاركت
\end{abstract}


قابلتوجهى بر روالهاى رايج روزمره خانواده داشته باشد [1 1 ].

والدين كودكان داراى اتيسم سطح بالاترى از افسردكى و

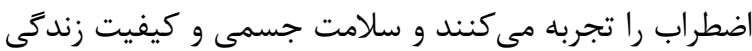

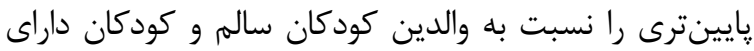

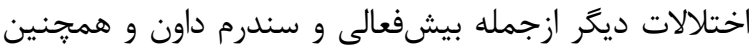

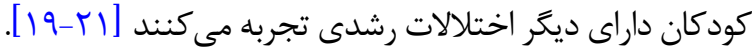

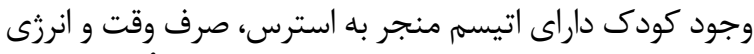

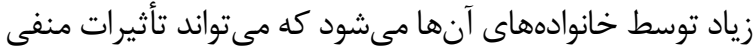

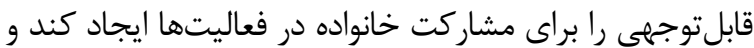

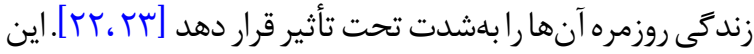

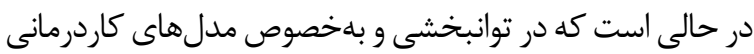

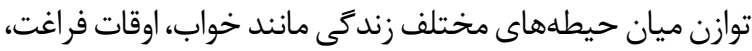

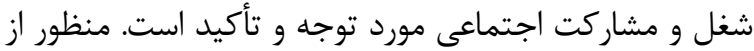

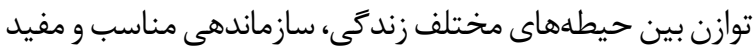

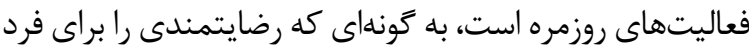

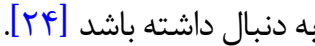

زبا توجه به افزايش شيوع اتيسم در سالهاى اخدير، ارتبان إنباط

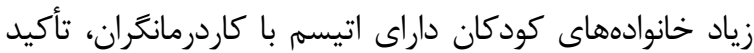

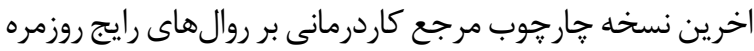

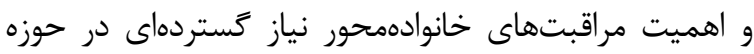

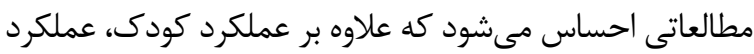

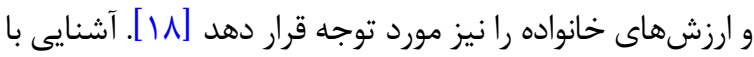

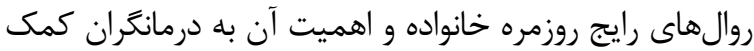

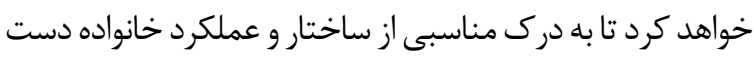

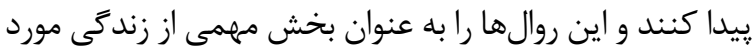

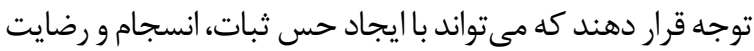

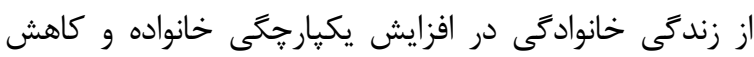

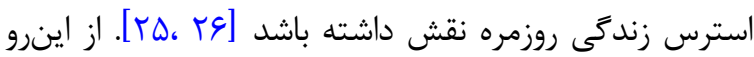

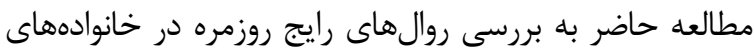
كودكان داراى اتيسم مى يردازد.

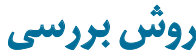

در اين يزوهش ابتدا در يايكاههاى اطلاعاتى-GubMed Sci Google Scholar g ence Direct جستوجو كه در بازه زمانى ابتداى تشكيل اين بايكاهها تا بايايان

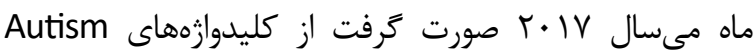
Partici- و و (Family ،Routines ، Daily Living ،Occupation

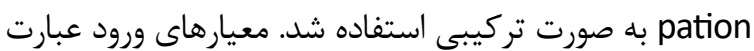

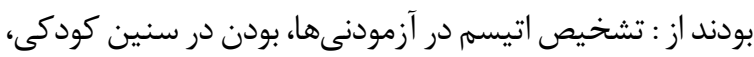
به زبان فارسى يا انغليسى بودن مقالات.

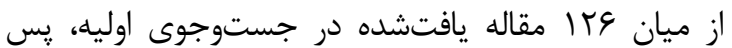

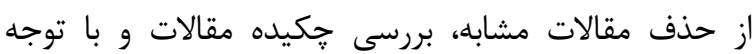

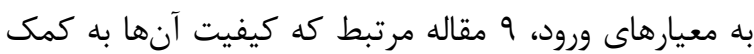

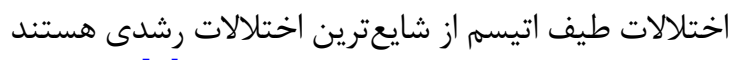

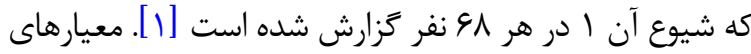

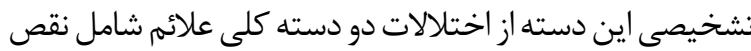

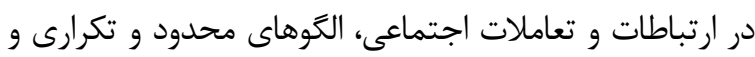

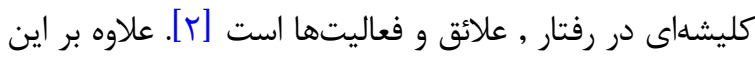

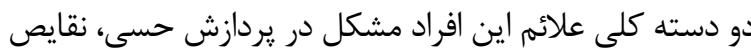

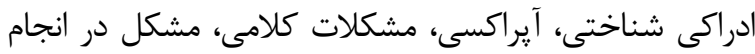

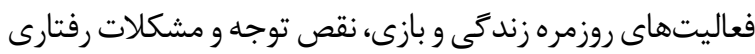

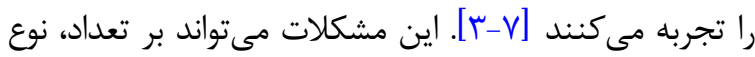

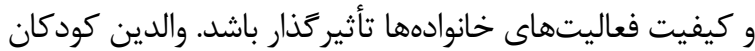

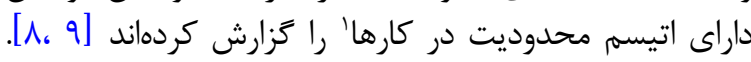

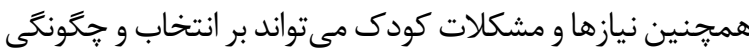

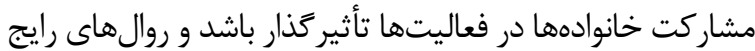

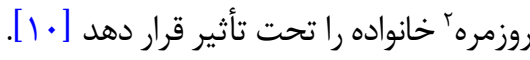

روالهاى رايج روزمره، الكوهاى تثبيتشده و منظمى از كارهاو

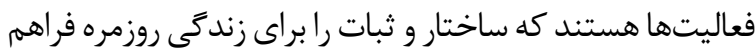

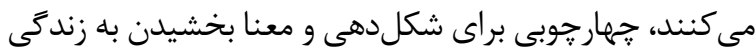

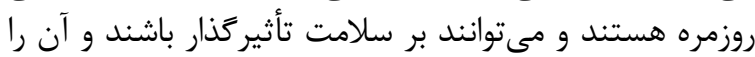

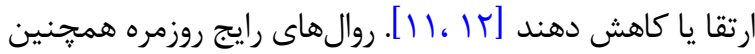

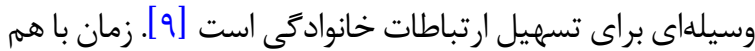

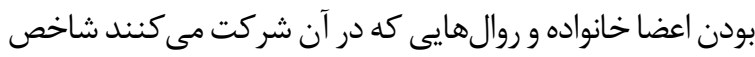

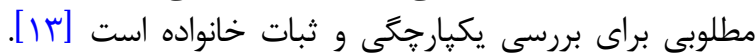

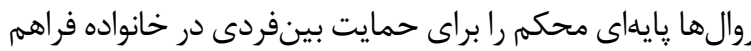

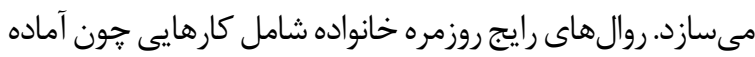

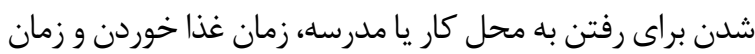

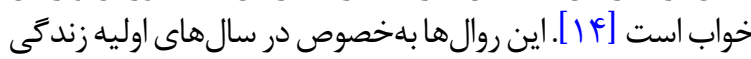

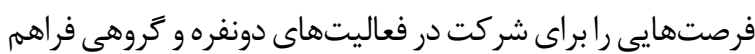

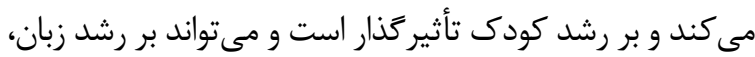

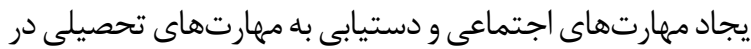

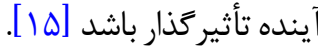

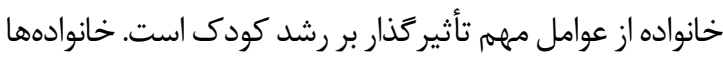

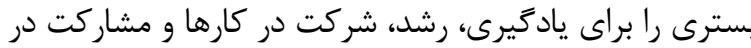

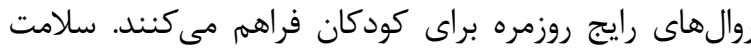

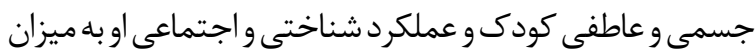

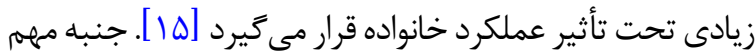

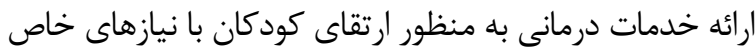

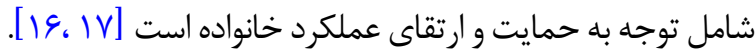

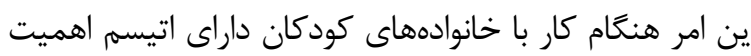

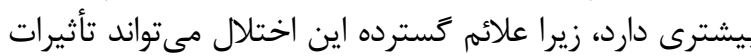

1. Occupation

2. Routines 


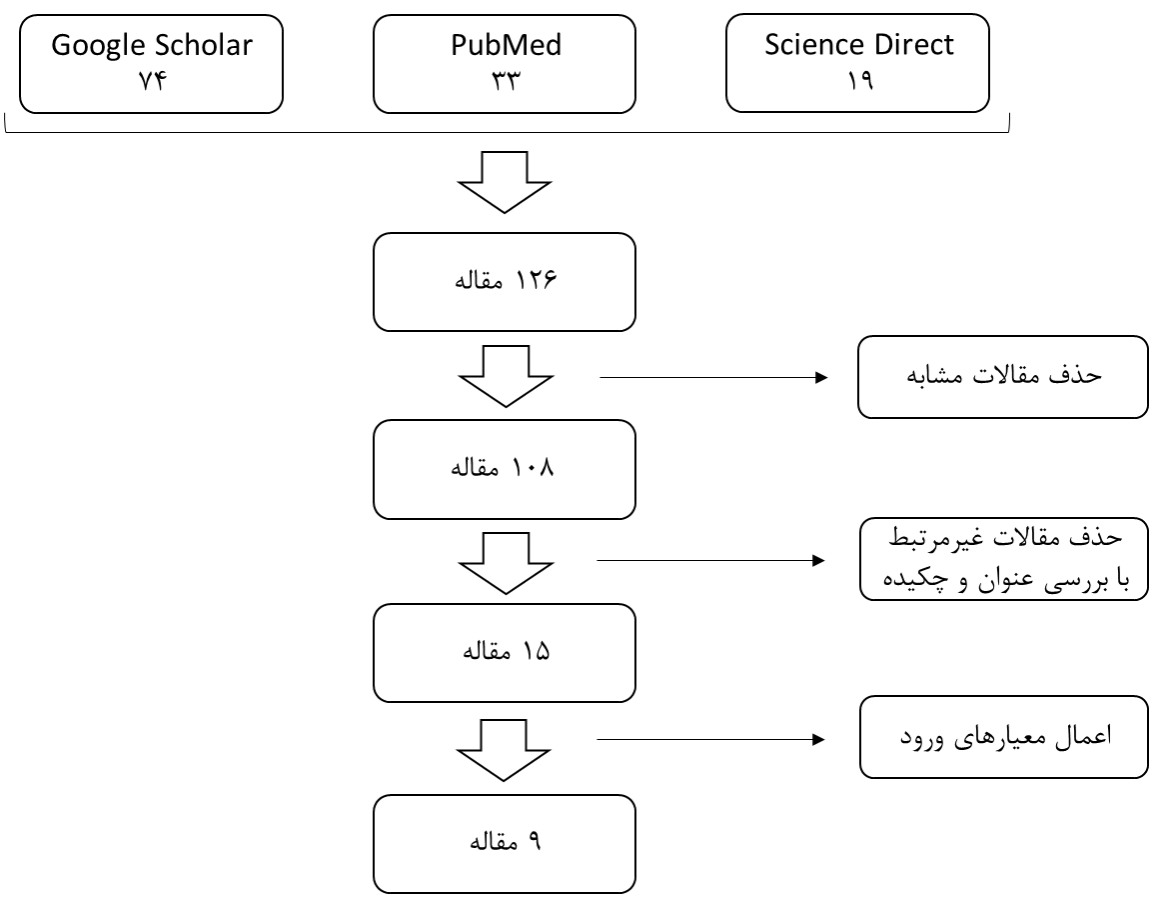

توانبخنتى

تصوير ا. جستوجو و كزينش مقالات

توجه قرار دهند كه در افزايش يكيارجگى خانواده و كاهش

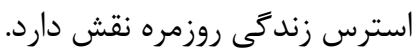

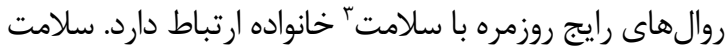

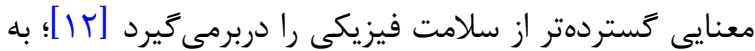

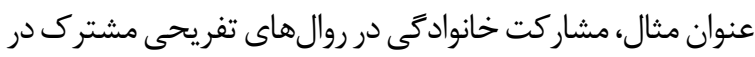
ارتقاى سلامت در خانوادههاى كودكان داراى معلوليت نقش دان دان دارد و فرصتهايى براى ايجاد حس شادمانى، عادى بودن و وكنترل

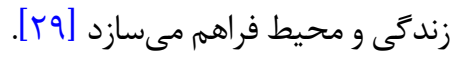

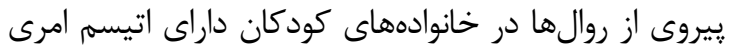

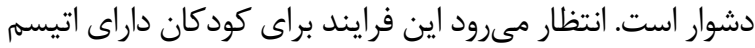

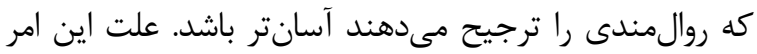

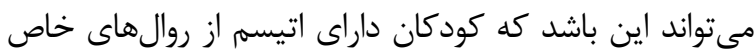

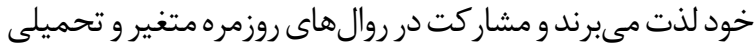

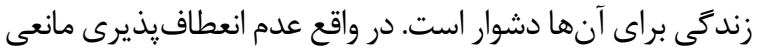

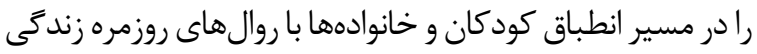

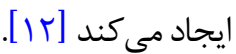

مطالعات صورت گرفته به اين اشاره دارند كه عواملى ازجمله

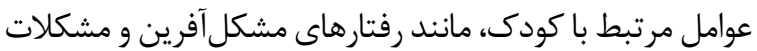

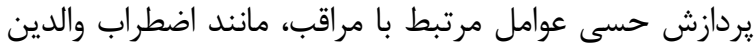

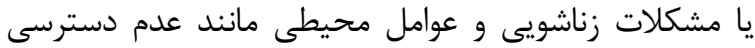

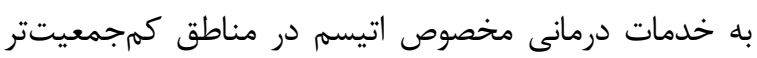

\section{Well-being}

ابزارهاى معتبر [YV، TN] ارزيابى و تأييد شد، به طور تمام متن

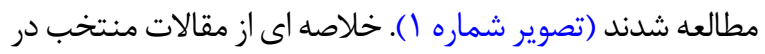

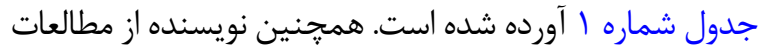
ديخر براى تكميل مطالب استفاده كرد.

LA

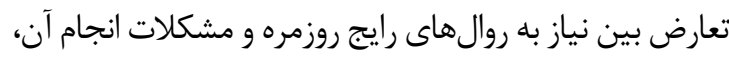

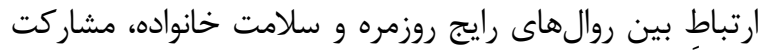

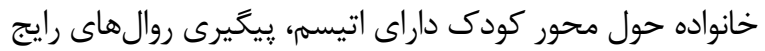

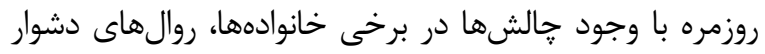

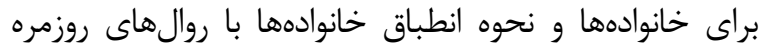

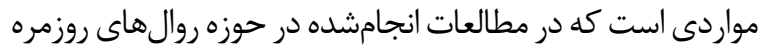

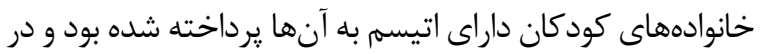

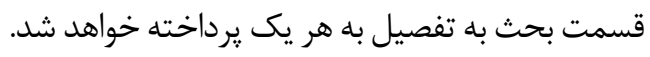

\section{tos}

آمارها نشان مى دهد شيوع اتيسم در سالهاى اخير افزايش

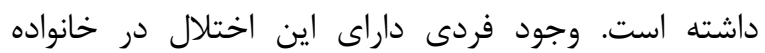

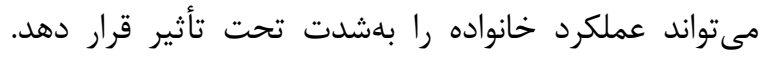

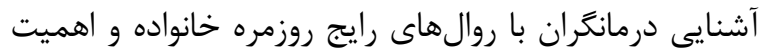

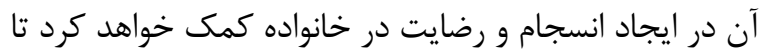

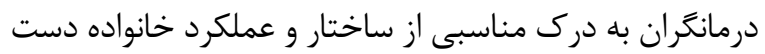

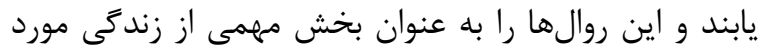


جدول ا. خلاصه مطالعات منتخب

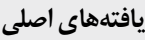

روش مطالعه

افراد مطالعهشده

سال

نويسنده

رديف

والدين در مناطق كمدر آمدتر ساعات اشتغال كمترى دارند و از طرفى دافى بايد

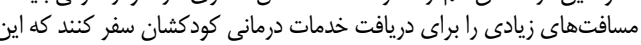

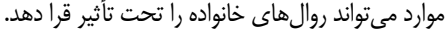

سطح بالاتر روالهاى خانوادگى با ميزان كيفيت زندگى بالاى خانواده ارتباط معنى دارى داشت.

\begin{tabular}{|c|c|}
\hline هrه نفر از والدين كودكان & مك اوليف' و همكاران \\
\hline
\end{tabular}

والدين زمان غذاخوردن رازمانى دشوار و استر سزا توصيف كردنا

والدين زمان عذا خوردن را زمانى دشوار و اسرسز اتوصيف كردند.

مسائل حسى كودكان در انتخاب فعاليتها توسط خانوادهها تأثير كذار است.

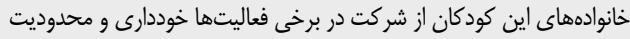

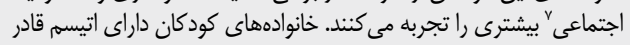

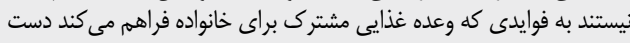
ياند.

\begin{tabular}{|c|c|c|c|c|}
\hline مقطعى & •داراى اتفر از والدين كودكان & $r+1 q$ & شلبوش [ُو همكاران & $r$ \\
\hline يديدارشناسى & داراى اتيسم از مادران كود آنان & $r \cdot 1 r^{c}$ & 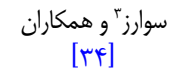 & r \\
\hline
\end{tabular}

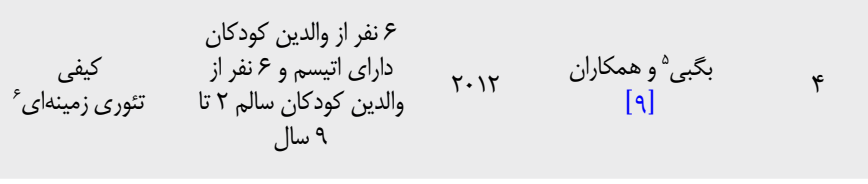

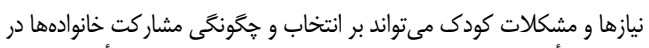

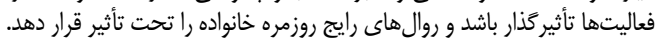

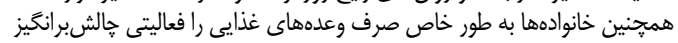
به شمار مى آورند.

\begin{tabular}{|c|c|c|c|c|}
\hline يديدارشناسى & 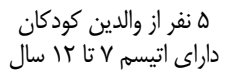 & $r .11$ & شاف و همكاران & $\Delta$ \\
\hline مقطعى & 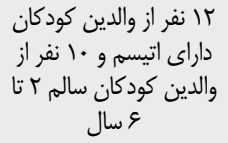 & $r+11$ & راجر و امايبالانج & 9 \\
\hline
\end{tabular}

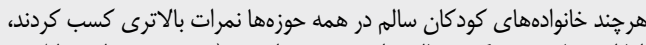

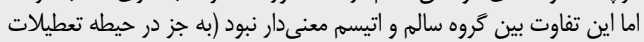

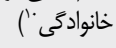

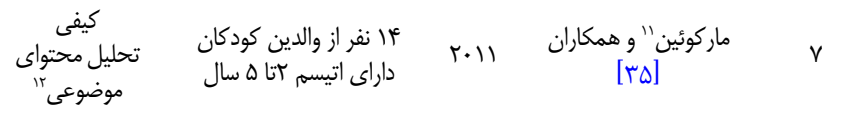

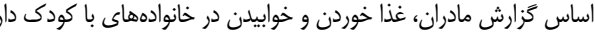

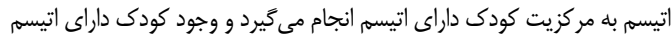

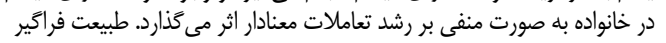

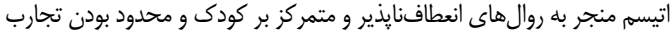

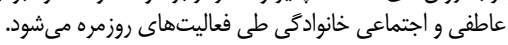

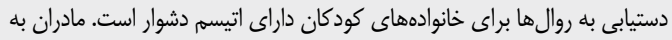

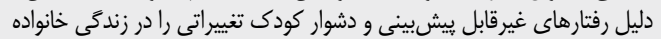

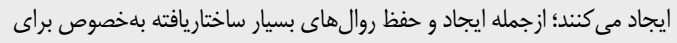

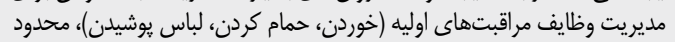

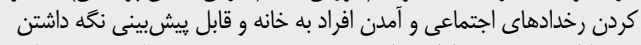

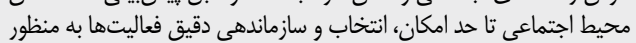

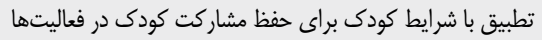

A

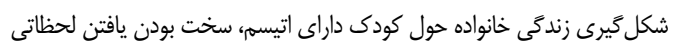

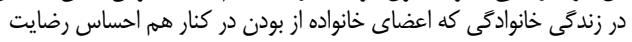
درونى داشته باشند.

1. McAuliffe

2. Schlebusch

3. Suarez

4. Phenomenological

5. Bagby

6. Grounded theory

7. Social limitation

و هويت خانواده نيز خواهد شد [Trا]. قرار كرفتن كودى داراى

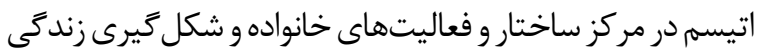

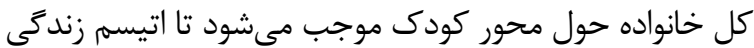

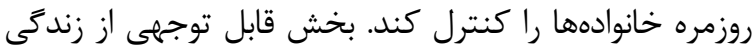

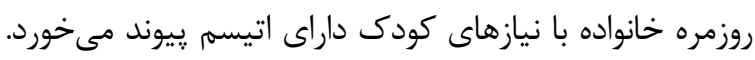

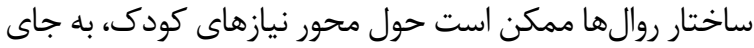
كل خانواده، شكل بكيرد و در برخى موارد نيازهاى ساير اعضاى نياى

\section{Schaaf}

9. Rodger \& Umaibalan

10. Family vacations

11. Marquenie

12. Thematic content analysis

13. Larson

14. DeGrace

و دورافتادتر عوامل توضيحدهنده دليل عدم توانايى والدين

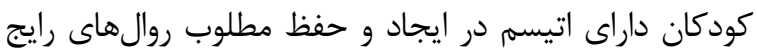

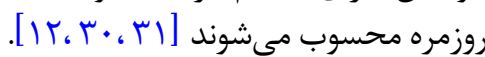

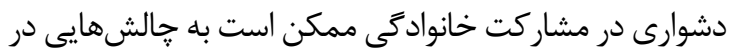

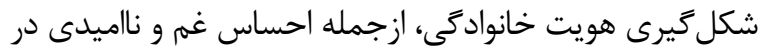

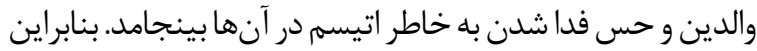
مشكل باروال هاى معنادار موجب بروز مسائلى در ارتباط باسلامت آنسايت 
خوابيدن ساختارمندتر و قابل پِيشبينىتر از غذا خوردن است

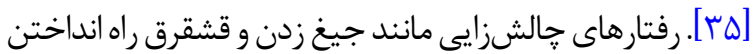

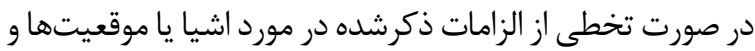

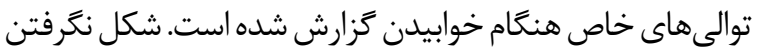

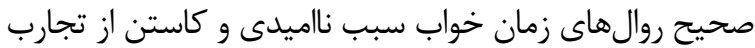

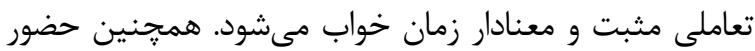

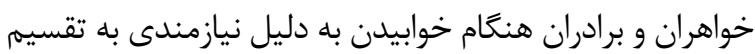

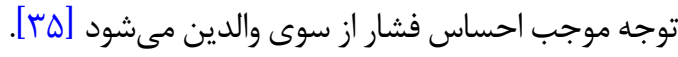
بر اساس كزارش مادران، غذا خوردن و خوابيدن در خانوادههاى

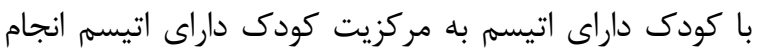

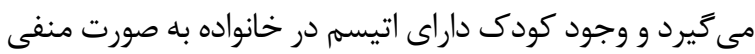

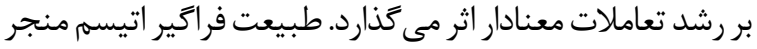

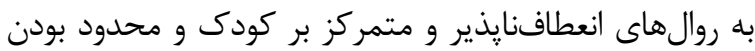

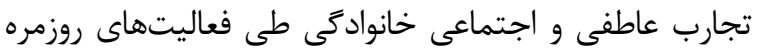

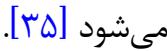

برخلاف آثار متمركز بر محدوديت مشاركت و دشوارى روالها،

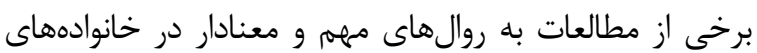

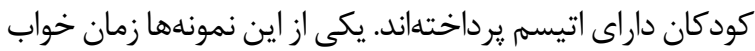

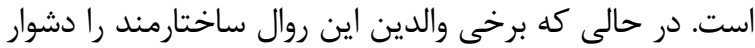

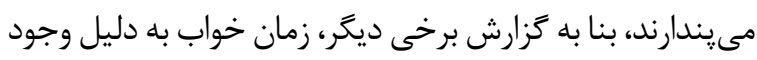

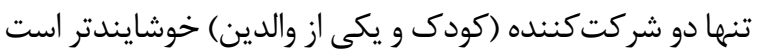

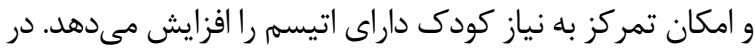

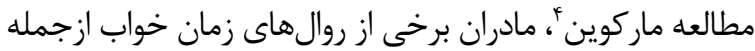

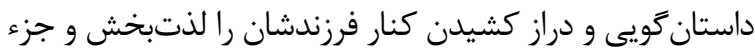

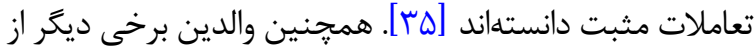

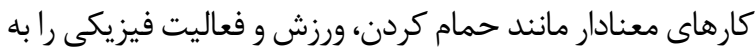

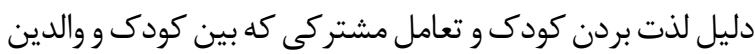

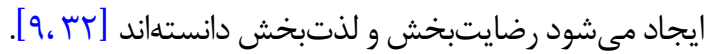

با وجود קالشهايى كه خانوادههاى كودكان داراى اتيسم

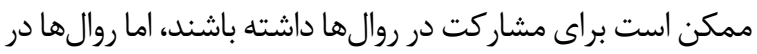

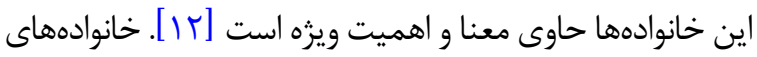

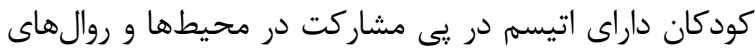

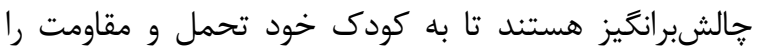

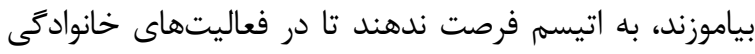

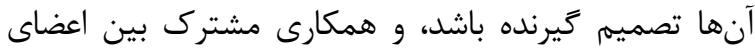

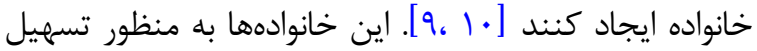

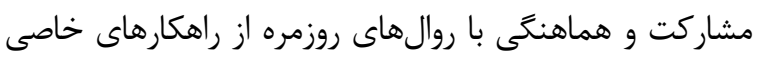

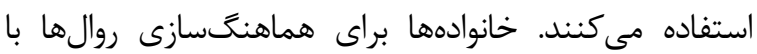

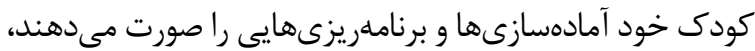

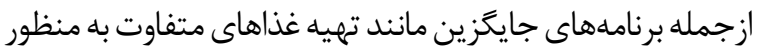

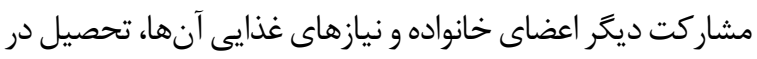

4. Marquenie

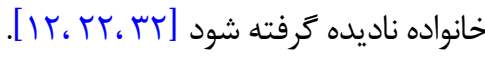
روالهاى خاص ازجمله صرف غذا و مشاركت در فعاليتهاى

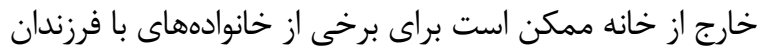

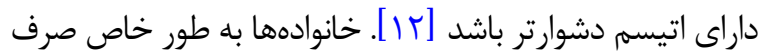

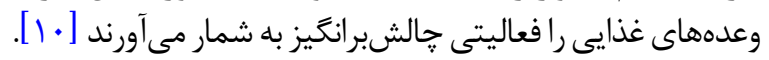

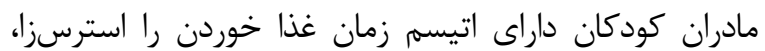

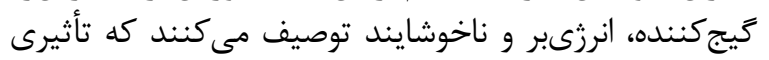

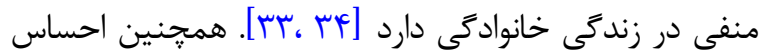

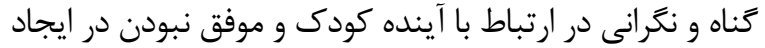

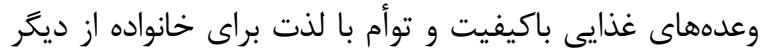

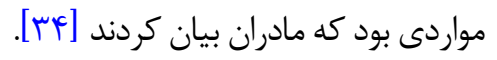

موارد ديخرى كه در اين رابطه كزارش شد شامل افزايش زمان

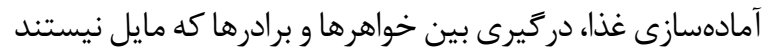

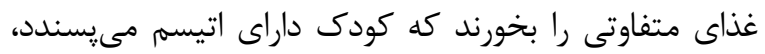

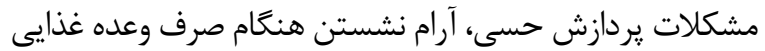

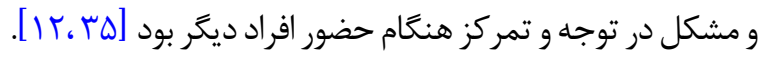
وعده غذايى يك كار مههم است و زمانى براى خانوادهها ارزشمند

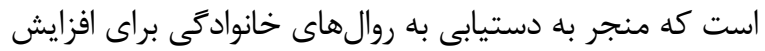

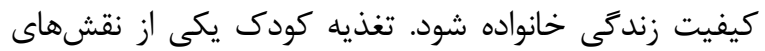

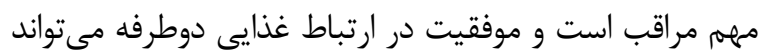

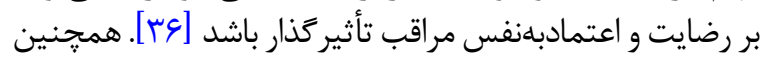

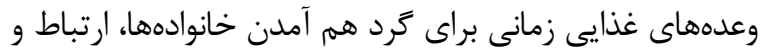

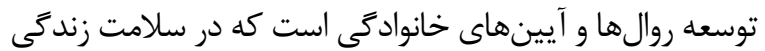

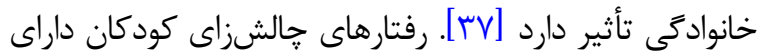

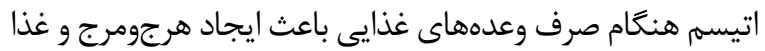

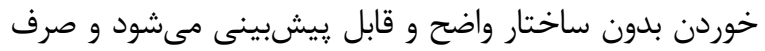

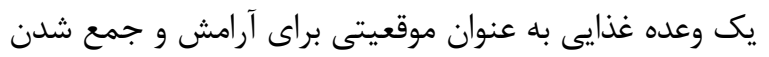

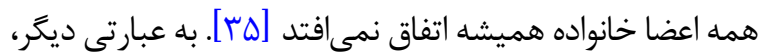

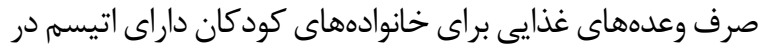

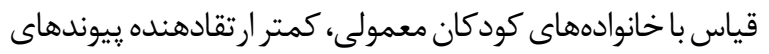

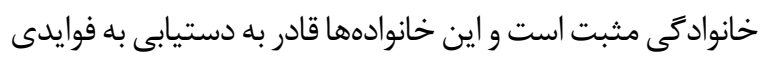

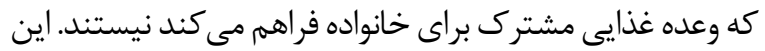

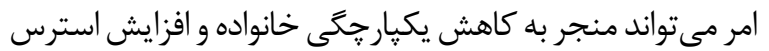

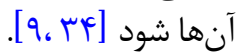

مادران كودكان داراى اتيسم زمان خواب را همم به عنوان زمانى

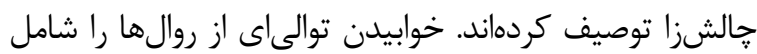

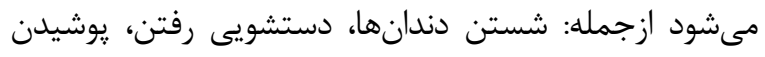

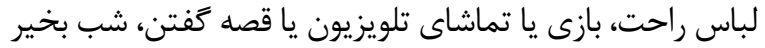

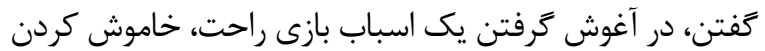

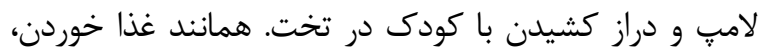

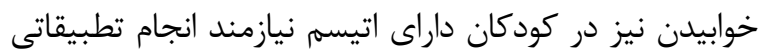

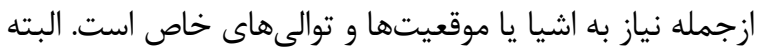


خانوادگى و ايجاد حس تعلق بين اعضاى خانواده، به نظر مىرسد

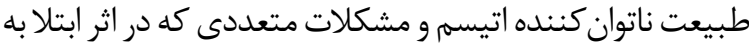

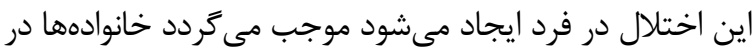

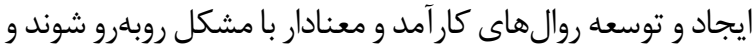

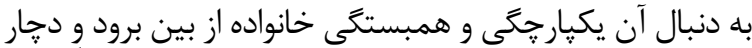

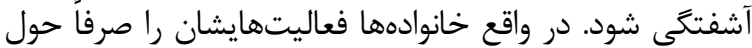
نيازها و مشكلات كودى داراى اتيسم خود برنامه ريزى مى كنئند.

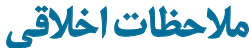

\section{ييروى از الصول الخاق يثوهش}

همه اصول اخلاقى در اين مقاله رعايت شده است. شركت كنند

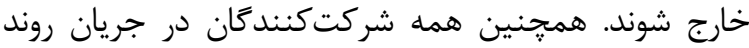

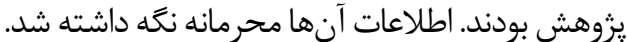

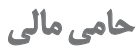

اين مقاله از يايان نامه كارشناسى ارشد آقاى احسان جمشيديان

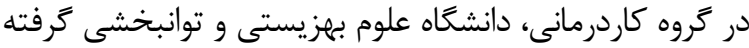

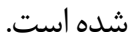

$$
\text { ت تعارض منافُ }
$$

بنابر اظهار نويسندگان اين مقاله تعارض منافع ندارد.

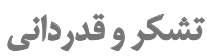

نويسند

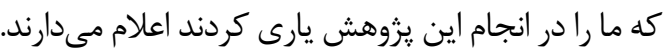

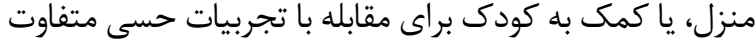

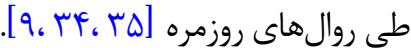

خانوادهها ممكن است در برنامههاى خود تغييراتى ايجاد كنند؛

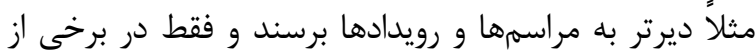

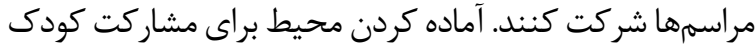

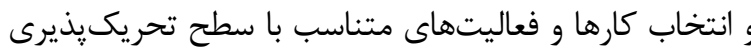
و ترجيحات كودك نيز از ديخر راهكارهايى است كه خانوادهها

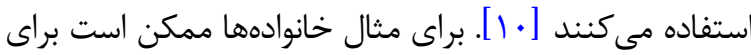
حفظ آرامش اقدام به صرف جداكانه وعدههاى غذايى آند كنند،

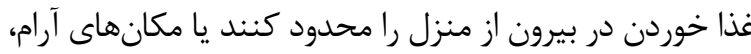

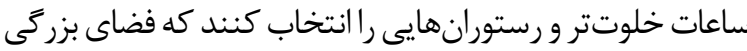
براى كودك دارند [باع].

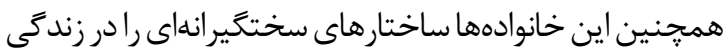

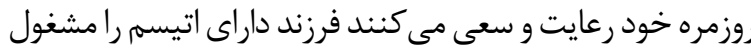

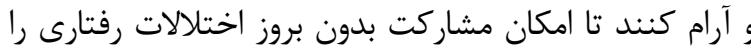

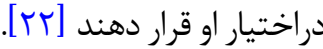

\section{نكات كاربردى براي درمانئران}

روالهاى رايج روزمره خانواده، بسترى براى كودكان با سن

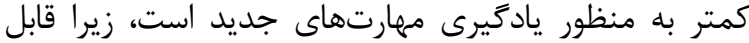

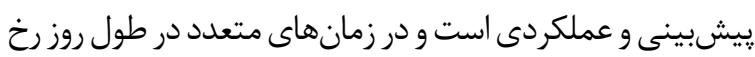

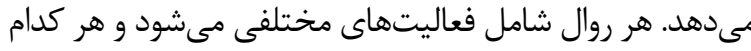

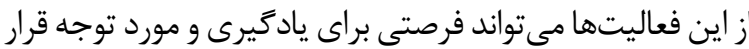

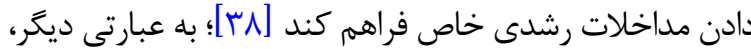

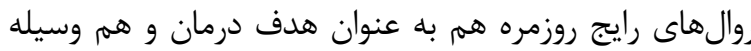

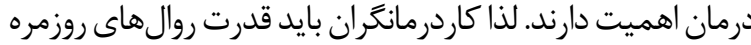

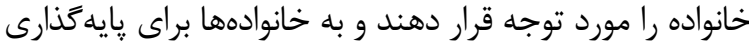

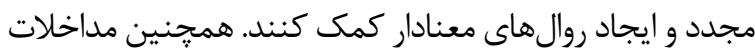

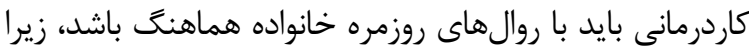

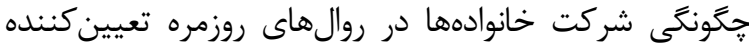

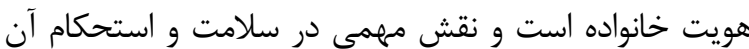

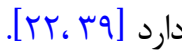

در مطالعات انجامشده عمدتاً به محدوديتهاى خانوادههاى

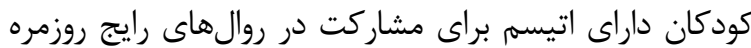

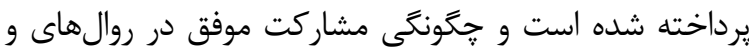

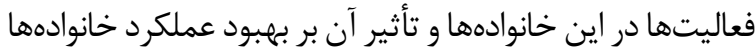
تبيين نشده است.

\section{5,5 areng}

با وجود نقش مهمم روالهاى رايج روزمره در رشد كودى و

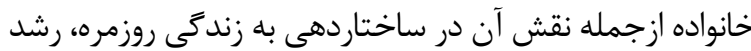

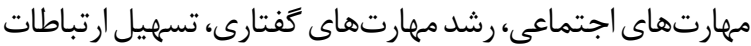




\section{References}

[1] Center for Disease Control and Prevention. Autism spectrum disorders (ASDs) data \& statistics [Internet]. 2017 [Updated 2018 April 26]. Available from: http://www.cdc.gov/ncbddd/autism/ data.html

[2] American Psychiatric Association. Diagnostic and Statistical Manual of mental disorders: DSM-5. Washington D.C.: American Psychiatric Association; 2013.

[3] Shiri V, Hosseini SA, Pishyareh E, Nejati V, Biglarian A. [Study the relationship of executive functions with behavioral symptoms in children with high-functioning autism (Persian)]. Archives of Rehabilitation. 2015; 16(3):208-17.

[4] Humphry R. Young children's occupations: Explicating the dynamics of developmental processes. American Journal of Occupational Therapy. 2002; 56(2):171-9. [DOI:10.5014/ ajot.56.2.171] [PMID]

[5] Parham LD, Mailloux Z. Sensory integration. In: Case-Smith J, O'Brien J, editors. Occupational Therapy For Children. Toronto: Mosby; 2010.

[6] Cosbey J, Johnston SS, Dunn ML. Sensory processing disorders and social participation. American Journal of Occupational Therapy. 2010; 64(3):462-73. [DOI:10.5014/ajot.2010.09076] [PMID]

[7] Jamshidian E, Jalili N, Haghgoo H. [The effect of sensory processing abilities on participation of children with autism (Persian)]. Daneshvar Medicine. 2016; 23(120):33-44.

[8] Baranek GT, Boyd BA, Poe MD, David FJ, Watson LR. Hyperresponsive sensory patterns in young children with autism, developmental delay, and typical development. American Journal on Mental Retardation. 2007; 112(4):233-45. [DOI:10.1352/08958017(2007)112[233:HSPIYC]2.0.CO;2]

[9] Bagby MS, Dickie VA, Baranek GT. How sensory experiences of children with and without autism affect family occupations. American Journal of Occupational Therapy. 2012; 66(1):78-86. [DOI:10.5014/ajot.2012.000604] [PMID] [PMCID]

[10] Schaaf RC, Toth-Cohen S, Johnson SL, Outten G, Benevides TW. The everyday routines of families of children with autism: Examining the impact of sensory processing difficulties on the family. Autism. 2011; 15(3):373-89. [DOI:10.1177/1362361310386505] [PMID]

[11] American Occupational Therapy Association (AOTA). Occupational therapy practice framework: Domain and process. American Journal of Occupational Therapy. 2014; 68:1-48. [DOI:10.5014/ajot.2014.682006]

[12] Boyd BA, McCarty CH, Sethi C. Families of children with autism: A synthesis of family routines literature. Journal of Occupational Science. 2014; 21(3):322-33. [DOI:10.1080/144275 91.2014.908816

[13] Brown O, Fouche P, Coetzee M. Bouncing forward: Families living with a type I diabetic child. South African Family Practice. 2010; 52(6):536-41. [DOI:10.1080/20786204.2010.10874044]

[14] Rodger S, Umaibalan V. The routines and rituals of families of typically developing children compared with families of children with autism spectrum disorder: An exploratory study. British
Journal of Occupational Therapy. 2011; 74(1):20-6. [DOI:10.42 76/030802211X12947686093567]

[15] Spagnola M, Fiese BH. Family routines and rituals: A context for development in the lives of young children. Infants \& Young Children. 2007; 20(4):284-99. [DOI:10.1097/01. IYC.0000290352.32170.5a]

[16] Raeis-Dana M, Tabatabaei-Nia M, Kamali M, Shafaroudi N. [From diagnosis to coping: a journey with parents in the course of the disability of their children (Persian)]. Archives of Rehabilitation. 2009; 10(1):42-51.

[17] Nazer M, Riyahi N, Mokhtaree M. [The effect of stress management training with cognitive behavioral style on stress and mental health of parents of children with intellectual disabilities (Persian)]. Archives of Rehabilitation. 2016; 17(1):32-41. [DOI:10.20286/jrehab-170130]

[18] Kuhaneck HM, Madonna S, Novak A, Pearson E. Effectiveness of interventions for children with autism spectrum disorder and their parents: A systematic review of family outcomes. American Journal of Occupational Therapy. 2015; 69(5):1-14. [DOI:10.5014/ajot.2015.017855] [PMID]

[19] Schieve LA, Blumberg SJ, Rice C, Visser SN, Boyle C. The relationship between autism and parenting stress. Pediatrics. 2007; 119(Supplement 1):S114-S21. [DOI:10.1542/peds.2006-2089Q] [PMID]

[20] Abbeduto L, Seltzer MM, Shattuck P, Krauss MW, Orsmond G, Murphy MM. Psychological well-being and coping in mothers of youths with autism, down syndrome, orfragile X syndrome. American Journal on Mental Retardation. 2004; 109(3):237-54. [DOI:10.1352/0895-8017(2004)1092.0.CO;2]

[21] Lee LC, Harrington RA, Louie BB, Newschaffer CJ. Children with autism: Quality of life and parental concerns. Journal of Autism and Developmental Disorders. 2008; 38(6):1147-60. [DOI:10.1007/s10803-007-0491-0] [PMID]

[22] DeGrace BW. The everyday occupation of families with children with autism. American Journal of Occupational Therapy. 2004; 58(5):543-50. [DOI:10.5014/ajot.58.5.543] [PMID]

[23] Lyons AM, Leon SC, Phelps CER, Dunleavy AM. The impact of child symptom severity on stress among parents of children with ASD: The moderating role of coping styles. Journal of Child and Family Studies. 2010; 19(4):516-24. [DOI:10.1007/s10826009-9323-5]

[24] Mohammadpour M, Rassafiani M, Ahmadi KM, Behnia F, Haghgoo HA, Biglarian A. Comparing the time-use of mothers with autistic children with that of mothers with healthy ones. Journal of Research in Rehabilitation Sciences. 2014; 10(1):182-92.

[25] Fiese BH, Tomcho TJ, Douglas M, Josephs K, Poltrock S, Baker $\mathrm{T}$. A review of 50 years of research on naturally occurring family routines and rituals: Cause for celebration. Journal of Family Psychology. 2002; 16(4):381-90. [DOI:10.1037/08933200.16.4.381] [PMID]

[26] Jensen EW, James SA, Boyce WT, Hartnett SA. The family routines inventory: Development and validation. Social Science \& Medicine. 1983; 17(4):201-11. [DOI:10.1016/02779536(83)90117-X] 
[27] Von Elm E, Altman DG, Egger M, Pocock SJ, Gøtzsche PC, Vandenbroucke JP. The Strengthening the Reporting of Observational Studies in Epidemiology [STROBE] statement: Guidelines for reporting observational studies. Gaceta Sanitaria. 2008; 22(2):144-50. [DOI:10.1157/13119325] [PMID]

[28] Long AF, Godfrey M. An evaluation tool to assess the quality of qualitative research studies. International Journal of Social Research Methodology. 2004; 7(2):181-96. [DOI:10.1080/1364557 032000045302]

[29] Downs ML. Leisure routines: Parents and children with disability sharing occupation. Journal of Occupational Science. 2008; 15(2):105-10. [DOI:10.1080/14427591.2008.9686616]

[30] McAuliffe T, Vaz S, Falkmer T, Cordier R. A comparison of families of children with autism spectrum disorders in family daily routines, service usage, and stress levels by regionality. Developmental Neurorehabilitation. 2017; 20(8):483-90. [DOI:10.10 80/17518423.2016.1236844] [PMID]

[31] Pourhidar M, Dadkhah A. The Effects of Individual and Group Training on General Health and Stress of Parents of Children with Autism Spectrum Disorders. Iranian Rehabilitation Journal. 2015;13(4):110-5.

[32] Larson E. Caregiving and autism: How does children's propensity for routinization influence participation in family activities? OTJR: Occupation, Participation and Health. 2006; 26(2):69-79. [DOI:10.1177/153944920602600205]

[33] Rogers LG, Magill-Evans J, Rempel GR. Mothers' challenges in feeding their children with autism spectrum disorder: Managing more than just picky eating. Journal of Developmental and Physical Disabilities. 2012; 24(1):19-33. [DOI:10.1007/s10882011-9252-2]

[34] Suarez MA, Atchison BJ, Lagerwey M. Phenomenological examination of the mealtime experience for mothers of children with autism and food selectivity. American Journal of Occupational Therapy. 2014; 68(1):102-7. [DOI:10.5014/ajot.2014.008748] [PMID]

[35] Marquenie K, Rodger S, Mangohig K, Cronin A. Dinnertime and bedtime routines and rituals in families with a young child with an autism spectrum disorder. Australian Occupational Therapy Journal. 2011; 58(3):145-54. [DOI:10.1111/j.14401630.2010.00896.x] [PMID]

[36] Thorne SE, Radford MJ, McCormick J. The multiple meanings of long-term gastrostomy in children with severe disability. Journal of Pediatric Nursing. 1997; 12(2):89-99. [DOI:10.1016/ S0882-5963(97)80029-2]

[37] Fulkerson JA, Story M, Neumark-Sztainer D, Rydell S. Family meals: Perceptions of benefits and challenges among parents of 8to 10 year old children. Journal of the American Dietetic Association. 2008; 108(4):706-9. [DOI:10.1016/j.jada.2008.01.005] [PMID]

[38] Woods J, Kashinath S, Goldstein H. Effects of embedding caregiver-implemented teaching strategies in daily routines on children's communication outcomes. Journal of Early Intervention. 2004; 26(3):175-93. [DOI:10.1177/105381510402600302]

[39] Schlebusch L, Samuels AE, Dada S. South African families raising children with autism spectrum disorders: Relationship be- tween family routines, cognitive appraisal and family quality of life. Journal of Intellectual Disability Research. 2016; 60(5):41223. [DOI:10.1111/jir.12292] [PMID] 
